\title{
The measurements of temperature and deformations of car radiators
}

\author{
Katarzyna Peta $^{1, *}$, and Karol Grochalski ${ }^{1}$ \\ ${ }^{1}$ Poznan University of Technology, Institute of Mechanical Technology, Piotrowo 3, 60-965 Poznan, Poland
}

\begin{abstract}
In the study main factors influencing the exploitative durability of the aluminum radiators used in motorization were classified. Special attention was put to thermal strains occurring during the usage of a car. The causes of theirs formation were identified, including disproportionate distribution of temperature in the construction of radiator, which changes cyclically depending on the characteristics of cooling fluid flow. In order to evaluate the influence of temperature and deformations of radiators on their durability, resistance tensometry method was used supplemented with temperature measurements with the use of thermoelements. Three linear resistive tensometers and three thermoelements were placed in the key areas of radiator (the inlet and outlet of cooling liquid to the heat exchanger and separator of the areas). Measurements were carried out during the examination of the durability of radiators on thermal shocks, which is one of the most basic examinations that imitates conditions of their work and verifies mechanical durability of products. Critical areas in the radiator were located, which are the most vulnerable to damages, including cracks. After the conducted research measurements of tightness were carried out, which verification is one of the most important requirements set for products in contact with intermediary medium in heat exchange. The study was supplemented with the observation of metallographic structures of the areas of fatigue cracks.
\end{abstract}

\section{Introduction}

Radiators used in the engine cooling system have to meet a number of exploitative requirements resulting from the conditions of their work, of which the most important are tightness, thermal efficiency, mechanical durability (endurance) and corrosion resistance. They are exposed mainly to activity of the medium fluid intermediary in heat exchange with rapidly changing temperature of the range from $-20^{\circ} \mathrm{C}$ to $100^{\circ} \mathrm{C}$ and the pressure of about 1 bar. Therefore, resistance to thermal shocks is the basic durability criterion of the radiators, which determines the construction of choices, production technology, the precision of making parts and the quality of their assembly [1-4].

With the increase of the time of exploitation of heat exchangers sectional plastic deformations develop, mainly brazed joints which consequently lead to crack initiations in these areas. The primary cause of their occurrence is thermal overloads, which intensity and caused results directly relate to working conditions of radiators in the engine cooling system. The main consequence of receiving and giving heat by radiator is increase or decrease of its temperature which leads to changes in linear measurements of the product according to the phenomenon of thermal expansion of materials. The limited possibility of deformation of construction results in the appearance of sectional heat strains usually caused by uneven distribution of temperature in radiator, inhomogeneity of mechanical properties, chemical composition and the metallographic structure of materials, especially brazed joints. The continuous activity of heat loads results in the thermal fatigue of radiator characterized by the net of cracks on its surface [5-11].

The determination of the degree of construction deformation, especially using nondestructive measurements methods is important in the assessments of its exploitative fatigue, in particular in the conditions of thermal loads. This group includes mainly tensometry methods and the methods of temperature measurements with the use of thermoelements. On this basis it is possible not only to determine the deformations of radiator but also to predict the location of possible cracks, which is important in industrial practice [12-17].

\section{Research methodology}

The measurements of temperature and distortions were carried out on car radiators made of clad aluminum tapes based on alloy of aluminum-manganese AA3003 (tape core material) and aluminum-silicon alloy (AA4343) (brazed material). The latter begins to melt at a temperature of $582^{\circ} \mathrm{C}$ and after the crystallization it forms inseparable joints in the heat exchanger. The chemical composition of the materials used in the productions of radiators are shown in Table 1 , their basic mechanical properties in Table 2 .

\footnotetext{
Corresponding author: katarzyna.peta@put.poznan.pl
} 
Table 1. The chemical composition limits of aluminum alloys used in the study.

\begin{tabular}{|c||c|c|c|c|c|c|c|}
\hline Alloy & Fe & Si & $\mathbf{C u}$ & $\mathbf{M g}$ & $\mathbf{M n}$ & $\mathbf{Z n}$ & $\mathbf{A l}$ \\
\hline \hline AA3003 & 0.7 & 0.6 & $\begin{array}{c}0.05- \\
0.2\end{array}$ & 0.05 & $\begin{array}{c}1.0- \\
1.5\end{array}$ & 0.1 & rem. \\
\hline AA4343 & 0.8 & $\begin{array}{c}6.8- \\
8.2\end{array}$ & 0.25 & 0.05 & 0.1 & 0.2 & rem. \\
\hline
\end{tabular}

Table 2. Basic mechanical properties of aluminum alloys used in the study.

\begin{tabular}{|c||c|c|c|}
\hline Material & $\begin{array}{c}\text { Tensile strength } \\
(\mathbf{R m}) \\
{\left[\mathbf{N} / \mathbf{m m}^{2}\right]}\end{array}$ & $\begin{array}{c}\text { Yield point } \\
\left(\mathbf{R p}_{\mathbf{0}, 2}\right) \\
{\left[\mathbf{N} / \mathbf{m m}^{2}\right]}\end{array}$ & $\begin{array}{c}\text { Elongation } \\
\left(\mathbf{A}_{\mathbf{5 0}}\right) \\
{[\mathbf{\%}]}\end{array}$ \\
\hline \hline $\begin{array}{c}\text { AA4343 4\% } \\
(\mathrm{clad})\end{array}$ & 115.00 & 58.00 & 37.00 \\
AA3003 & & & \\
\hline
\end{tabular}

Brazing is the main operations of the manufacturing process of radiators influencing the formation of inseparable joints and their durability. They were carried out in the protective atmosphere of nitrogen in a radiantconvective furnace, which main areas included:

- thermal degreasing (the removal of processing oils),

- application of non-corrosive flux,

- heating in a convective chamber,

- brazing in a radiant chamber,

- cooling in the protective atmosphere of nitrogen,

- cooling in atmosphere air.

The flow of cooling fluid in the examined radiator from its inlet to outfall proceeded in the shape of "U" letter, according to the diagram in Fig. 1. The direction of the circulation of cooling fluid has a significant effect on the distribution of temperature in the heat exchanger, consequently distribution of deformations.

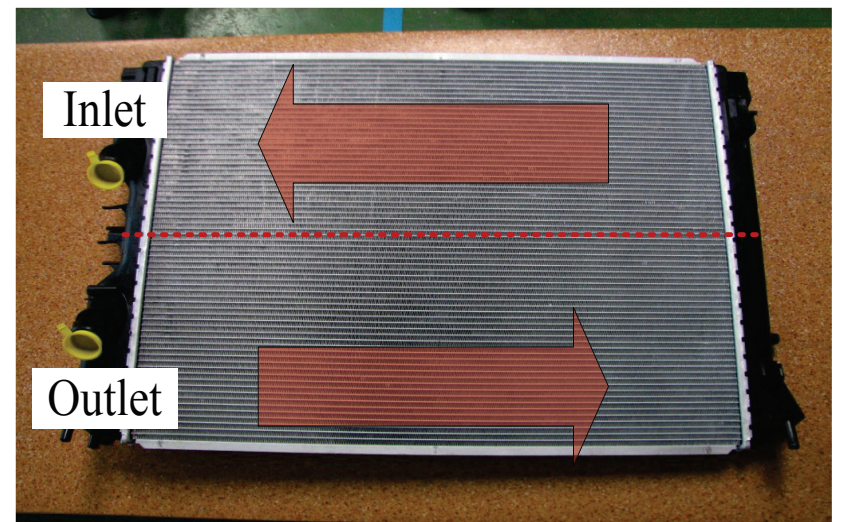

Fig. 1. The view of circulation of cooling fluid through radiator.

The examination of endurance of radiators consisted of temperature measurements and deformations in environment imitating the conditions of their work. For this purpose, endurance test of radiators on activity of thermal shocks was performed, based on the circulation of cooling liquid (aqueous solution of ethylene glycol) through the heat exchanger which the temperature-time cycle included (Fig. 2 and Fig. 3):

- the increase of temperature of cooling liquid from $20^{\circ} \mathrm{C}$ to $100^{\circ} \mathrm{C}$ within $30 \mathrm{~s}$,

- the stabilization of cooling liquid of $100^{\circ} \mathrm{C}$ within $30 \mathrm{~s}$,

- the decrease of temperature of cooling liquid from $100^{\circ} \mathrm{C}$ to $-20^{\circ} \mathrm{C}$ within $30 \mathrm{~s}$,

- the stabilization of cooling liquid of $-20^{\circ} \mathrm{C}$ within $30 \mathrm{~s}$.

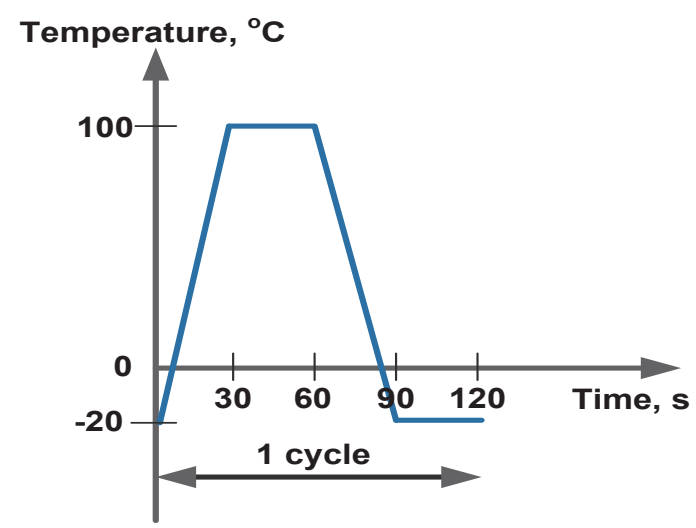

Fig. 2. The temperature-temporal profile of cooling liquid during the endurance examination of the heat exchanger on thermal shocks.

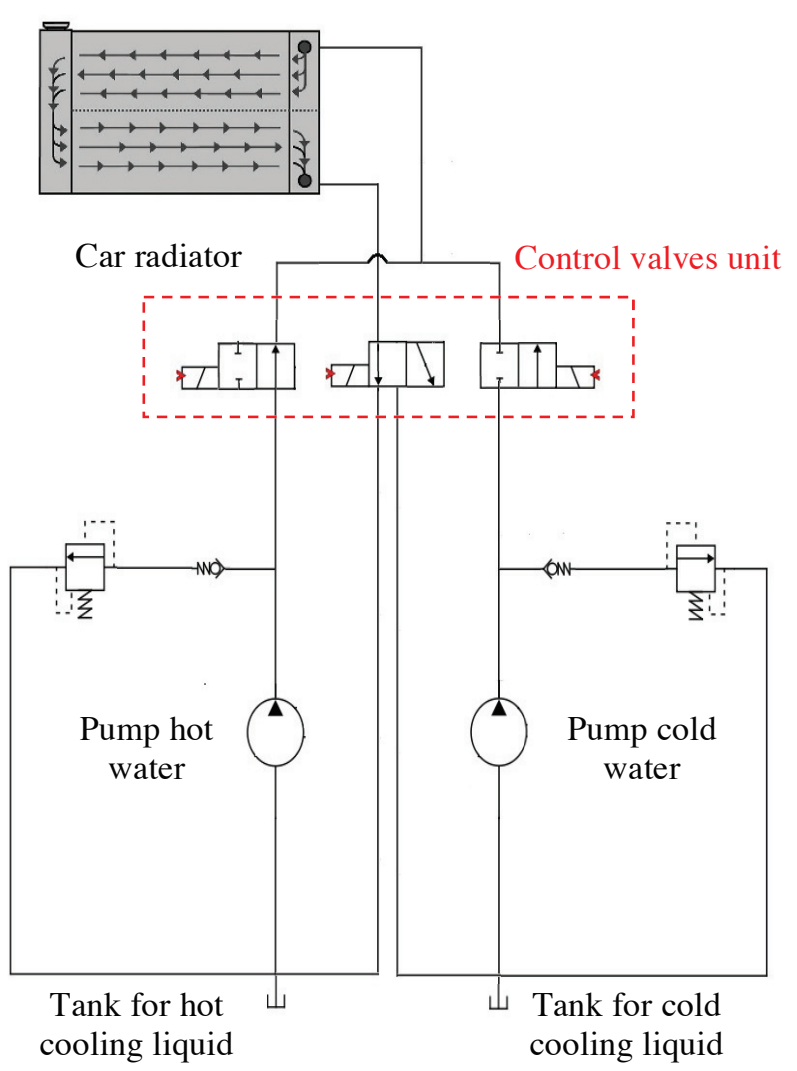

Fig. 3. The temperature-temporal profile of cooling liquid during the endurance examination of the heat exchanger on thermal shocks. 
The study was conducted in the temperature of environment of $23^{\circ} \mathrm{C}$ and the strength of the flow of cooling liquid was $4000 \mathrm{~kg} / \mathrm{h}$.

The measurements of temperatures and deformations of radiators were carried out during the endurance examination on activity of thermal shocks, which reflects the actual working conditions. For this purpose, tensometry method was used, which is based on the determination of tensometer deformations caused by acting on its external forces and temperature measurement method with the use of thermoelements. Three linear resistive tensometers were used with self-compensating properties of the nominal resistance of $120 \Omega \pm 0.35 \%$, gauge factor $2.09 \pm 1.0 \%$, transverse sensitivity $0.2 \%$ and temperature compensation modulus for aluminum of $\alpha=23 \times 10^{-6} / \mathrm{K}$. They were placed in the key areas of radiator, i.e. the inlet and outlet of cooling liquid to the heat exchanger and separator of the areas. In the same areas thermoelements were installed so as to determine dependence of the deformations of the heat exchanger from the temperature on its surface (Fig. 4). Thermoelements from NiCr$\mathrm{NiAl}$ alloy of $0.5 \mathrm{~mm}$ diameter and a length of $200 \mathrm{~mm}$ were used.

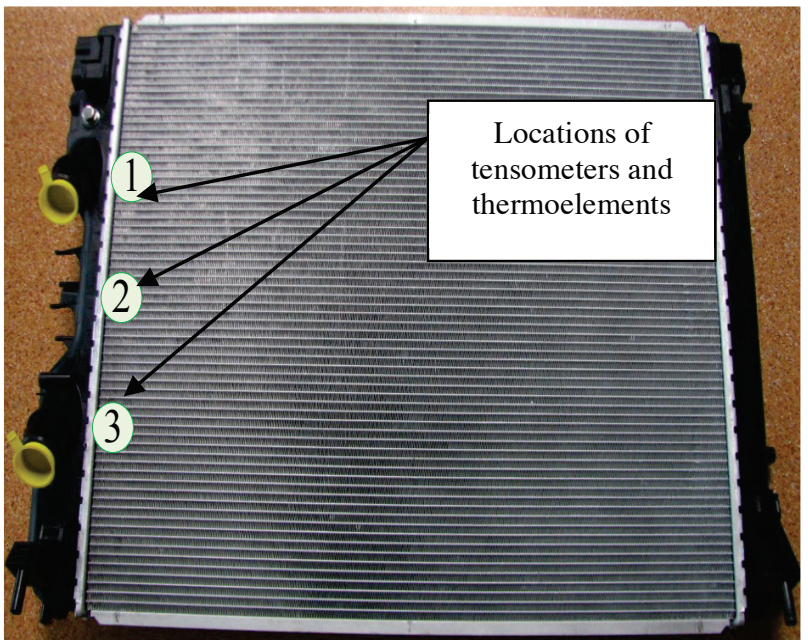

Fig. 4. The diagram of the locations of tensometers and thermoelements in radiator.

\section{Discussion of obtained results}

During the test of endurance of radiator on thermal shocks, the temperature and deformations measurements of the construction were carried out. The obtained results allowed to determine the effects of temperature changes of the radiator on the formation of deformations of its construction. It has been determined that with the increase of radiator temperature what follows is the extending of its deformations, thereby the change of linear measurements of the product. It is directly connected with the phenomenon of thermal expansion of materials.

The diagrams shown in Fig. 5, Fig. 6 and Fig. 7 present the profile of temperature dependence and deformations from the time for three considered areas in the radiator, which deformation changes largely in a linear degree with the change of its temperature which is related to the thermal conductance of aluminum. The effect of sectional concentration of strains and consequently the biggest changes of the linear measurements of construction occurs in temperature of cooling fluid of $100^{\circ} \mathrm{C}$, which is noticeable in the form of characteristic peak in the inserted diagrams. What was obtained is the repeatability of the results of temperature measurements and radiator deformations in the following cycles of measurements their endurance for thermal shocks. The greatest value of the deformations was recorded in the area of inlet of cooling fluid to radiator, which is associated with the occurrence of the biggest changes of temperature in this area, ranging from $-20^{\circ} \mathrm{C}$ to $100^{\circ} \mathrm{C}$. In the area of its outfall the intensity of the influence of thermal shocks on the radiator decreases because the heat of cooling liquid is gradually channeled by the heat exchanger. It determines a smaller thermal impact in this area of the radiator, resulting in smaller deformations of construction.

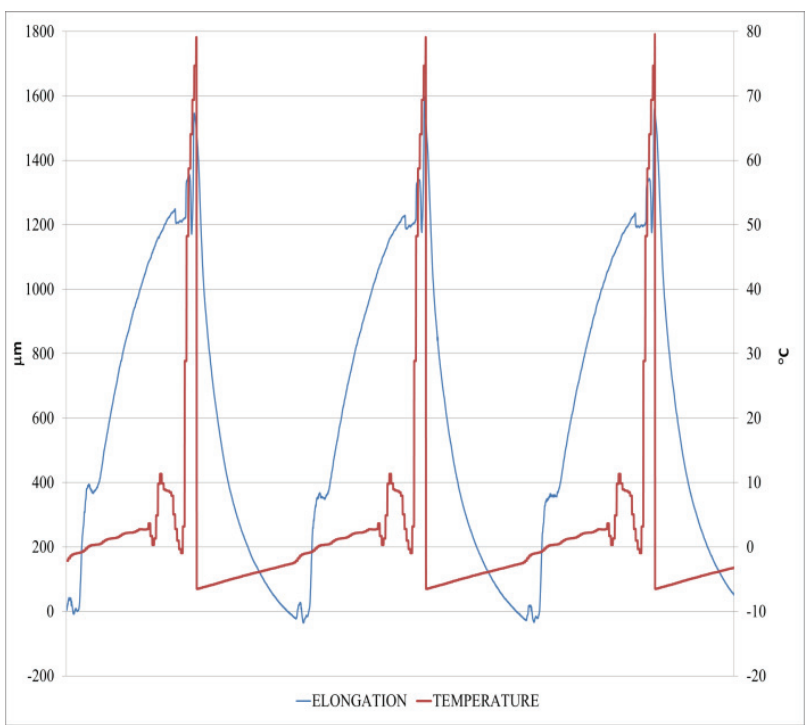

Fig. 5. The diagram of temperature and deformations in the function of time for the area of inlet of cooling liquid to the heat exchanger.

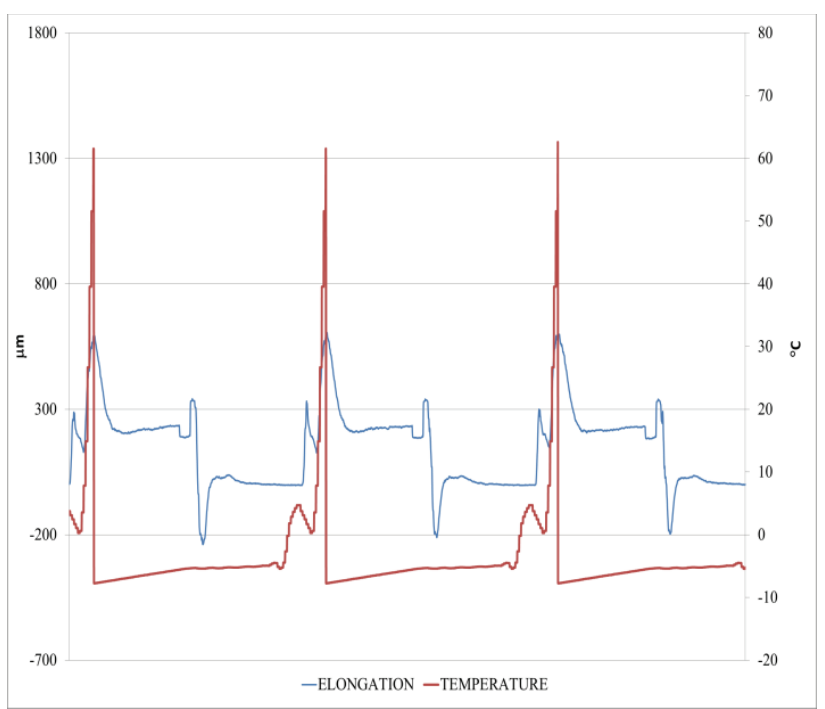

Fig. 6. The diagram of temperature and deformations in the function of time for in the area of separator of the inlet and outfall of cooling liquid areas. 
After the completion of 1500 measurements cycles in the endurance examination of the radiator on thermal shocks according to manufacturing requirements, the measurements of its tightness were carried out, which did not show the leak of cooling liquid.

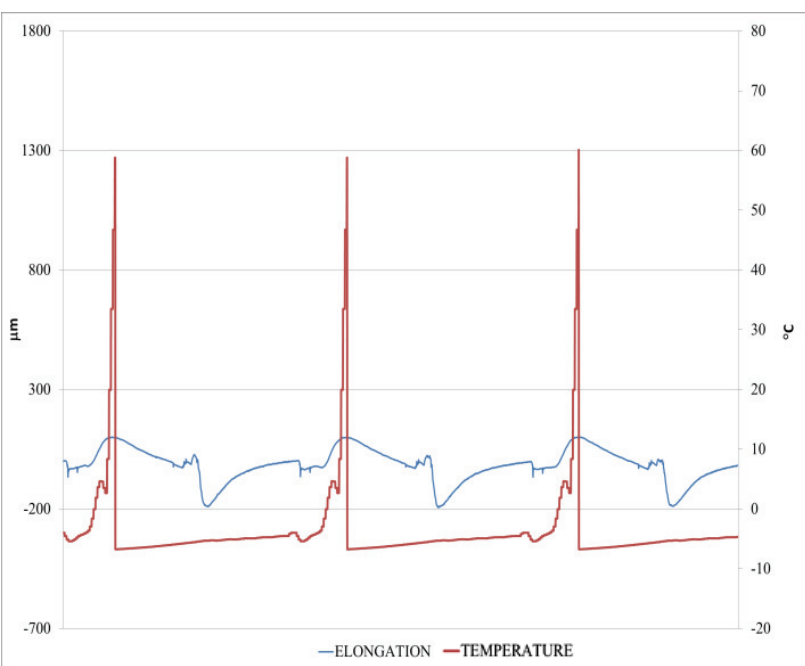

Fig. 7. The diagram of temperature and deformations in the function of time for outlet area of cooling liquid from the heat exchanger.

The heat exchanger fulfilled the established quality requirements. In order to identify the mechanism of forming of the permanent distortion of the contraction further 1500 cycles of endurance examination on thermal shocks were performed. The result of the prolonged exposures to cyclical thermal loads was weakening of the material, consequently the formation on fatigue cracks (Fig. 8). They appeared in the tube-tank joints area, mainly in zone of inlet of cooling liquid to radiator.

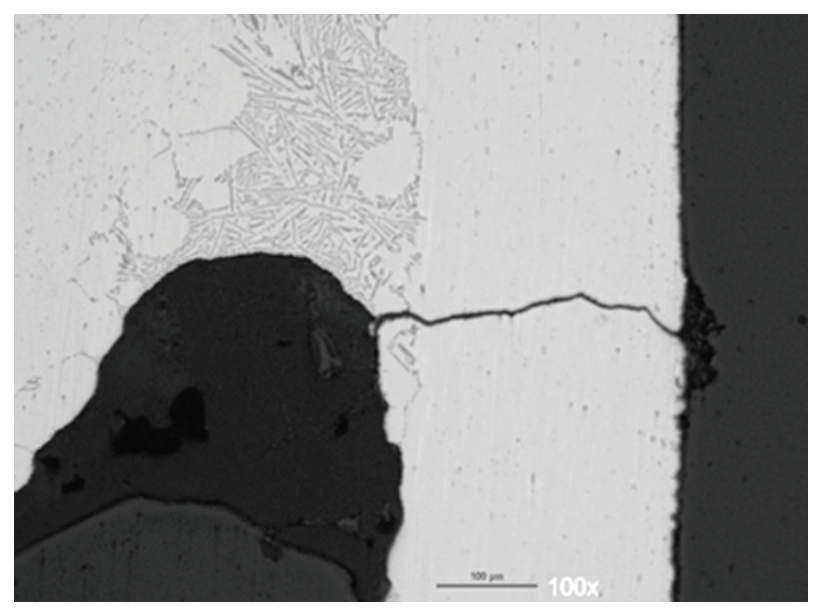

Fig. 8. The view of fatigue crack after the endurance examination of radiator of fluids on thermal shocks.

\section{Conclusions}

The most important final conclusions concerned the achieved results are:

- the effect of cooling liquid temperature was determined based on the distortions of radiators,
- uneven distribution of cooling liquid temperature values in different areas of the heat exchanger was observed,

- temperature measurements with the use of thermoelements were supplemented by tensometry measurements of distortions allowing to locate the areas of the radiator that are the most exposed to the effects of heat activity, which consequently leads to fatigue cracks,

- $\quad$ increased closing control of the heat exchangers should in particular focus on the area of inlet of the cooling fluid to the radiator, where there are the highest values of deformation during their exploitation,

- the obtained results are useful for industrial practice by indicating the method to identifying radiators construction deformations.

\section{References}

1. A. Mutai, Appl. Therm. Eng. 1 (2015)

2. R. Prakash., M. Selvam, A. Pandian, S. Palani, K. Harish, Indian J. Sci. Technol. 9 (2016)

3. K. Thulukkanam, Heat Exchanger Design Handbook. Second Edition (CRC Press, Boca Raton, 2013)

4. G. Prudhvi, G. Vinay, G. Babu, Int. J. Eng. Adv. Technol. 2 (2013)

5. J. Nowacki, M. Kawiak, Przegląd Spawalnictwa, 81 (2009)

6. H. Kim, S. Lee, J. Mech. Sci. Technol. 26 (2012)

7. A. Kłyszewski, J. Żelechowski, A. Frontczak, P. Rutecki, W. Szymański, Z. Zakotowicz, M. Nowak, Arch. Metall. Mater. 59 (2014)

8. K. Czapczyk, P. Siwak, P. Jabłoński, Ł. Furmański, P. Grobelny, S. Legutko, Adv. Sci. Technol. Res. J. 10 (2016)

9. A. Saigal, E. Fuller, Comp. Mater. Sci. 21 (2001).

10. S. Kahl, H. Ekström, J. Mendoza, Metall. Mater. Trans. A 45A (2014)

11. T. Miller, S. Adamczak, J. Świderski, M. Wieczorowski, A. Łętocha, B. Gapiński, B. Pol. Acad. Sci-Tech. 65 (2017)

12. Y. Chen, A.H. Clausen, O.S. Hopperstad, M. Langseth, Int. J. Solids Struct. 46 (2009)

13. G. Owolabi, D. Odoh, A. Peterson, A. Odesh, H. Whitworth, World J. Mech. 3 (2013)

14. T.S.B. Naser, G. Krallics, Acta Polytech. Hung. 11 (2014)

15. J. Maszybrocka, A. Stwora, B. Gapiński, G. Skrabalak, M. Karolus, B. Pol. Acad. Sci-Tech. 65 (2017)

16. N. Rossini, M. Dassisti, B. Khaled, O. Abdul, Mater. Design 35 (2012)

17. P. Jabłoński, P. Czajka, Proceedings of 5th International Conference on Advanced Manufacturing Engineering and Technologies. Belgrade-Serbia (2017) 